\title{
Aspirina versus rivaroxabana na prevenção do tromboembolismo venoso após artroplastia total do joelho: Um ensaio clínico randomizado, controlado e duplo-cego*
}

\section{Aspirin versus Rivaroxaban to Prevent Venous Thromboembolism after Total Knee Arthroplasty: A Double-blinded, Randomized Controlled Trial}

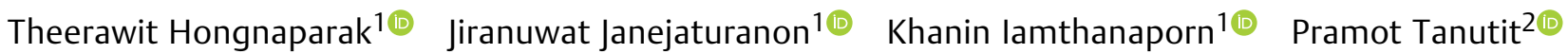
Varah Yuenyongviwat ${ }^{1}$

${ }^{1}$ Departamento de Ortopedia, Faculdade de Medicina, Universidade

Endereço para correspondência Varah Yuenyongviwat, MD, Prince of Songkla, Songkhla, Tailândia

2 Departamento de Radiologia, Faculdade de Medicina, Universidade Department of Orthopedics, School of Medicine, Prince of Songkla University, Hat Yai, Songkhla 90110, Tailândia

Prince of Songkla, Songkhla, Tailândia (e-mail: varahortho@gmail.com).

Rev Bras Ortop 2022;57(5):741-746.

\section{Resumo \\ Objetivo A aspirina (ácido acetilsalicílico, AAS) e a rivaroxabana são anticoagulantes que vêm ganhando popularidade devido à facilidade de uso na prevenção do tromboembolismo venoso (TEV) após artroplastia total do joelho (ATJ). Este estudo teve como objetivo avaliar a eficácia do AAS em comparação com a da rivaroxabana na profilaxia de TEV em pacientes submetidos a ATJ. \\ Método Quarenta pacientes com osteoartrite primária do joelho, que seriam sub- metidos a ATJ, foram randomizados em dois grupos. No total, 20 pacientes do grupo AAS usaram aspirina oral, na dose de $300 \mathrm{mg} /$ dia, para a profilaxia do TEV após ATJ; e 20 pacientes do grupo rivaroxabana receberam uma dose oral de $10 \mathrm{mg} /$ dia. No $4^{\circ}$ e $14^{\circ}$ \\ Palavras-chave \\ - aspirina \\ - rivaroxabana \\ - artroplastia do joelho \\ - tromboembolia venosa dias do pós-operatório, trombose venosa profunda (TVP) dos membros inferiores no lado da cirurgia foi detectada por meio de ultrassonografia duplex. Foram registradas outras complicações durante catorze dias. \\ Resultados Não foram detectados achados positivos de TVP com a ultrassonografia duplex nos grupos de pacientes, e não se observou a ocorrência de embolia pulmonar. No total, 4 pacientes apresentaram equimose subcutânea no $4^{\circ}$ dia do pós-operatório}

Estudo desenvolvido no Departamento de Ortopedia da Faculdade de Medicina da Universidade Prince of Songkla, Songkhla, Tailândia. recebido

25 de Janeiro de 2021

aceito após revisão em

14 de Maio de 2021

Publicado on-line

Outubro 25, 2021
DOI https://doi.org/

10.1055/s-0041-1735941. ISSN $0102-3616$. (c) 2021. Sociedade Brasileira de Ortopedia e Traumatologia. All rights reserved.

This is an open access article published by Thieme under the terms of the Creative Commons Attribution-NonDerivative-NonCommercial-License, permitting copying and reproduction so long as the original work is given appropriate credit. Contents may not be used for commercial purposes, or adapted, remixed, transformed or built upon. (https://creativecommons.org/ licenses/by-nc-nd/4.0/)

Thieme Revinter Publicações Ltda., Rua do Matoso 170, Rio de Janeiro, RJ, CEP 20270-135, Brazil 


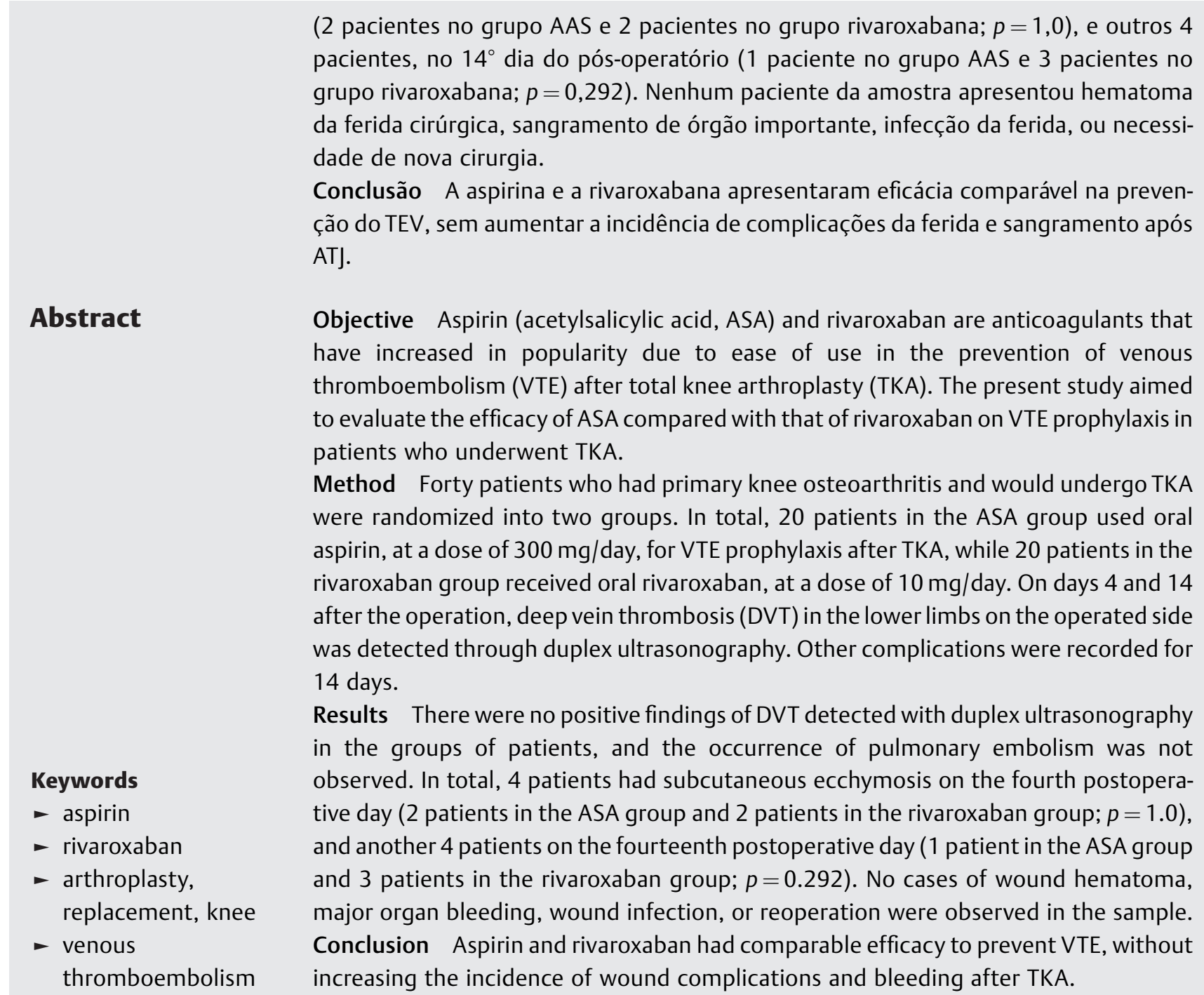

\section{Introdução}

O tromboembolismo venoso (TEV) é uma complicação que pode ocorrer após a artroplastia total do joelho (ATJ). ${ }^{1}$ Existem muitas modalidades para prevenir o TEV, como os dispositivos mecânicos, protocolo de deambulação precoce, e anticoagulantes. ${ }^{2}$ Atualmente, os anticoagulantes estão incluídos nos protocolos de tratamento padrão para quase todos os pacientes, exceto para aqueles que têm alguma contraindicação, como sangramento recente de órgão interno. ${ }^{3}$ Muitos anticoagulantes são prescritos para prevenir oTEV; entretanto, a aspirina (ácido acetilsalicílico, AAS) e a rivaroxabana são anticoagulantes que têm tido uma grande aceitação em virtude da facilidade de uso. ${ }^{4,5}$ Esses anticoagulantes vêm na forma oral, e não exigem um monitoramento da coagulação sanguínea. Além disso, o AAS e a rivaroxabana apresentam eficácia na prevenção do TEV após ATJ. ${ }^{6,7}$

A aspirina tem a vantagem de ter baixo custo e ser de fácil administração, e há um estudo ${ }^{8}$ que relata menor risco de complicações hemorrágicas com o seu uso, como hematoma de ferida operatória ou sangramento de órgão interno, em comparação com o uso de outros anticoagulantes. A rivaroxabana é um inibidor do fator Xa, com a vantagem de ser um potente anticoagulante e de fácil administração. ${ }^{9}$ Alguns estudos compararam a eficácia e a segurança do AAS e da rivaroxabana em pacientes submetidos a ATJ. Além disso, em um pequeno ensaio clínico randomizado e controlado, ${ }^{10}$ os autores constataram que não havia diferença na incidência de trombose venosa profunda (TVP) sintomática e complicações locais e sistêmicas entre os pacientes que receberam AAS e rivaroxabana. No entanto, em outro ensaio clínico randomizado e controlado, ${ }^{11}$ os autores relataram uma menor incidência de TVP em pacientes que receberam rivaroxabana em comparação com os que receberam AAS. ${ }^{11}$

Devido às limitações dos ensaios clínicos randomizados e controlados que comparam a eficácia do AAS e da rivaroxabana, combinadas com a falta de consenso quanto aos resultados dos estudos anteriores, este estudo teve como objetivo avaliar, em pacientes submetidos a ATJ, a eficácia do 
AAS em comparação com a rivaroxabana na profilaxia de TEV. Os autores levantaram a hipótese de que o AAS poderia prevenir a incidência do TEV após ATJ de forma tão eficaz quanto a rivaroxabana.

\section{Métodos}

Este ensaio clínico prospectivo, randomizado, e controlado foi realizado com um grupo dividido em 2 braços, com uma proporção de alocação de 1:1, em um ambiente hospitalar com alta tecnologia, no período de 10 de agosto de 2016 a 31 de dezembro de 2018. O estudo foi aprovado pelo comitê de ética e conselho de revisão institucional. O protocolo do estudo foi realizado de acordo com os princípios éticos da Declaração de Helsinque para pesquisas médicas envolvendo participantes humanos. O termo de consentimento livre e esclarecido foi assinado de forma individual por todos os participantes. Registro de ensaios clínicos (http://www.clinicaltrials.in.th): TCTR20201226005.
Ao todo, 47 pacientes com osteoartrite primária do joelho, com mais de 50 anos de idade e com indicação de ATJ, foram abordados para possível inclusão. Os critérios de exclusão foram pacientes com histórico de coagulopatias, de TEV, uso recente de anticoagulantes, azóis e inibidores de proteinase, antecedentes de alergia aos fármacos usados neste estudo, alergia ao material de contraste para a realização de tomografia computadorizada, doença renal crônica grave, cirrose e asma. No total, 7 pacientes foram excluídos do estudo: 2 pacientes tinham histórico de TEV, 2 tinham doença renal crônica, 1 tinha cirrose, e 2 se recusaram a participar do estudo. Por fim, 40 pacientes foram incluídos no estudo (-Fig. 1).

Os pacientes foram alocados em dois grupos: no grupo AAS, 20 pacientes receberam $300 \mathrm{mg}$ de AAS para a profilaxia de TEV, ao passo que, no grupo rivaroxabana, 20 pacientes receberam $10 \mathrm{mg}$ de rivaroxabana com o mesmo fim. Os pacientes de ambos os grupos tomaram os medicamentos uma vez ao dia, após o café da manhã, durante 14 dias, a

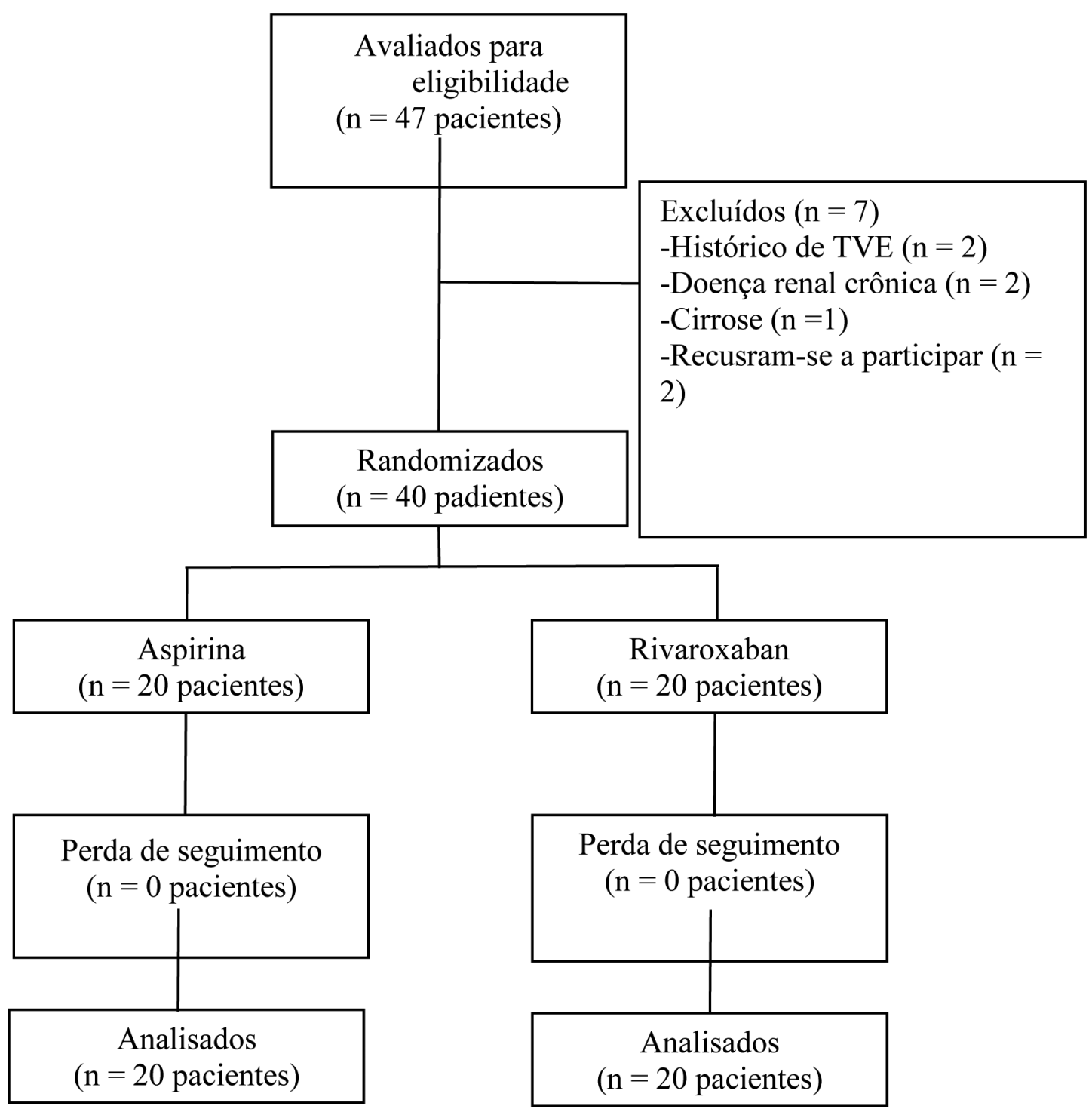

Fig. 1 Fluxograma do estudo. 
Tabela 1 Dados demográficos da amostra do estudo

\begin{tabular}{|l|l|l|l|}
\hline Característica & Grupo rivaroxabana: $\mathbf{n}=\mathbf{2 0}$ & Grupo ácido acetilsalicílico (AAS): $\mathbf{n}=\mathbf{2 0}$ & Valor de $\mathbf{p}$ \\
\hline Idade (anos) & $70,5 \pm 7,25^{*}$ & $68,15 \pm 7,43^{*}$ & 0,318 \\
\hline Gênero (masculino:feminino) & $3: 17$ & $7: 13$ & 0,144 \\
\hline Lado (direito:esquerdo) & $12: 8$ & $11: 9$ & 0,749 \\
\hline Índice de massa corporal $\left(\mathrm{kg} / \mathrm{m}^{2}\right.$ ) & $26,91 \pm 3,66^{*}$ & $29,15 \pm 4,49^{*}$ & 0,092 \\
\hline Hematócrito pré-operatório (\%) & $38,42 \pm 4,57$ & $38,41 \pm 4,13$ & 0,994 \\
\hline Plaquetas (células/mm ${ }^{3}$ ) & $272,350 \pm 106,355$ & $261,760 \pm 116,594$ & 0,766 \\
\hline Tempo do torniquete (minutos) & $117,6 \pm 39,22$ & $121,6 \pm 24,41$ & 0,701 \\
\hline Tempo cirúrgico (minutos) & $163,65 \pm 43,16$ & $167 \pm 29,84$ & 0,777 \\
\hline
\end{tabular}

Nota: *Médias e desvios padrão.

partir do dia seguinte à ATJ. Todos os pacientes foram submetidos à cirurgia de prótese total do joelho cimentada com estabilização posterior, com o emprego da mesma técnica cirúrgica, mediante torniquete pneumático inflado durante toda a cirurgia. Cefazolina foi usada na profilaxia da infecção cirúrgica. No caso de alergias a penicilina ou a cefalosporina, foi usado o fármaco clindamicina. Utilizouse um dreno de sucção de sistema fechado antes do fechamento da ferida, que foi removido 48 horas após a cirurgia. Os pacientes foram autorizados a realizar exercícios isométricos de quadríceps e bombeamento de tornozelo imediatamente após a operação, e iniciaram uma série de exercícios de movimento e deambulação com um dispositivo de apoio no dia seguinte à cirurgia.

O hematócrito foi dosado 6 horas após a cirurgia, assim como no $4^{\circ}$ e $14^{\circ}$ dias de pós-operatório. No $4^{\circ}$ e $14^{\circ}$ dias de pós-operatório, um radiologista musculoesquelético experiente realizou o diagnóstico de TVP dos membros inferiores foi realizado por meio de ultrassonografia duplex. No caso de algum paciente apresentar qualquer suspeita clínica de embolia pulmonar, fez-se uma tomografia computadorizada em espiral do tórax, a fim de confirmar o diagnóstico. As ocorrências pós-operatórias, como complicações da ferida e sangramento inesperado, foram acompanhadas atentamente durante 14 dias após a cirurgia.

Os pacientes foram alocados com randomização em blocos de quatro, por meio de uma sequência numérica aleatória gerada por computador, que foi depositada em envelopes opacos lacrados. $O$ envelope foi aberto na manhã do dia da cirurgia por um farmacêutico na farmácia do ambulatório. Os pacientes, o médico e o avaliador desconheciam os grupos de pacientes.

\section{Análise Estatística}

Para a análise dos dados, usou-se o programa R(R Foundation for Statistical Computing, Viena, Áustria), versão 3.1.0. Por meio do teste $t$, foram avaliados os dados demográficos dos pacientes, como idade, índice de massa corporal (IMC), hematócrito pré e pós-operatórios, contagem de plaquetas, e o tempo de aplicação do torniquete e o tempo cirúrgico (em minutos). 0 teste do qui-quadrado de Pearson foi utilizado para comparar gênero, lado, taxa de transfusão de sangue, incidência de TVP, embolia pulmonar, complicação da ferida cirúrgica, sangramento, e outras ocorrências. A significância estatística foi assumida com valores de $p$ menores do que 0,05 .

\section{Resultados}

As características demográficas, incluindo idade, gênero, lado operado, IMC, hematócrito pré-operatório, contagem de plaquetas, tempo de aplicação do torniquete, e o tempo cirúrgico não foram significativamente diferentes entre os dois grupos $(p>0,05)$ ( - Tabela $\mathbf{1}$ ).

Não foram detectados achados positivos de TVP com a ultrassonografia duplex em ambos os grupos, e tampouco se observou a ocorrência de pacientes com sintomas de embolia pulmonar. $\mathrm{O}$ hematócrito pós-operatório não foi diferente entre os grupos após 6 horas (AAS: 33,69 $\pm 4,01 \%$; rivaroxabana: $32,79 \pm 3,48 \% ; p=0,453$ ), 24 horas (AAS: $31,33 \pm 3,3 \%$; rivaroxabana: $29,93 \pm 2,99 \% ; p=0,17$ ), e 2 semanas (AAS: $34,33 \pm 4,75 \%$; rivaroxabana: $33,75 \pm 3,07 \% ; p=0,649)$ do pós-operatório. Cinco pacientes no grupo AAS e seis no grupo rivaroxabana receberam transfusões de sangue alogênico $(p=0,723)$. Ao todo, 4 pacientes apresentaram equimoses subcutâneas no $4^{\circ}$ dia do pós-operatório ( 2 pacientes no grupo AAS e 2 no grupo rivaroxabana; $p=1,0$ ), e outros 4 , no $14^{\circ}$ dia do pós-operatório (1 paciente no grupo AAS e 3 pacientes no grupo rivaroxabana; $p=0,292$ ). Na amostra, não houve pacientes com hematoma da ferida, sangramento de órgão importante, infecção da ferida, ou nova cirurgia.

\section{Discussão}

A aspirina e a rivaroxabana são amplamente utilizadas na prevenção de TEV após ATJ. Estudos anteriores ${ }^{12-14}$ que compararam a eficácia e a segurança do AAS e da rivaroxabana demonstraram não haver diferença nas taxas de TEV, complicações hemorrágicas, complicações da ferida, e reinternação hospitalar. No entanto, um estudo retrospectivo ${ }^{15}$ relatou que pacientes que fizeram uso do AAS apresentaram um risco maior de desenvolver TEV, embolia pulmonar, e reinternação.

Os autores decidiram realizar o presente estudo em virtude das limitações dos ensaios clínicos randomizados e 
controlados que compararam o AAS e a rivaroxabana na prevenção do TEV após ATJ, e dos estudos anteriores em que não houve consenso quanto aos resultados. Nossos resultados demonstraram que o uso de AAS ou rivaroxabana não acarreta diferenças na incidência de TEV, de TVP na triagem por ultrassonografia duplex, e de embolia pulmonar, e nas taxas de transfusão de sangue, risco de sangramento, e complicações da ferida cirúrgica.

Este estudo apresenta algumas limitações. Em primeiro lugar, o número limitado de participantes, que poderia ser insuficiente para detectar diferenças com relação ao TEV. Em segundo lugar, havia uma proporção maior de pacientes do sexo feminino acima do peso pelos cálculos do IMC. Por fim, todos os pacientes do estudo eram de etnia asiática, que apresentam uma taxa menor de TEV em comparação com as populações ocidentais. ${ }^{16}$ No entanto, acreditamos que nossos achados poderiam ser aplicados a pacientes ocidentais e do sexo masculino, assim como aos que se encontram em outras faixas do IMC.

Este estudo demonstrou que não houve diferença nas incidências de TEV, e de TVP na triagem por ultrassonografia duplex; também não houve diferença com relação à embolia pulmonare nos dois grupos de pacientes após ATJ. Nossos resultados são apoiados por um estudo do Brasil. Colleoni et al. ${ }^{10}$ realizaram um pequeno ensaio clínico randomizado, com 32 pacientes, para comparar doses de $300 \mathrm{mg}$ de AAS com dose de $20 \mathrm{mg}$ de rivaroxabana, e não observaram diferença na taxa de TVP entre os grupos. No entanto, um ensaio clínico randomizado, controlado e prospectivo anterior contradiz esses resultados: Zou et al. ${ }^{11}$ relataram que pacientes que usaram $10 \mathrm{mg} /$ dia de rivaroxabana tiveram uma menor incidência de TVP, do que os pacientes que usaram $100 \mathrm{mg} /$ dia de AAS oral. Nossa hipótese é a de que a dose menor de AAS no estudo de Zou et al. ${ }^{11}$ pode ter sido um fator que contribuiu para um resultado diferente tanto do presente estudo quanto do estudo de Colleoni et al. ${ }^{10}$

Quanto às complicações do uso da medicação, este estudo demonstrou complicações menores com o uso de AAS e de rivaroxabana: equimoses subcutâneas pós-operatórias, que não diferiram em termos de incidência entre os grupos. Não houve complicações importantes, como infecção da ferida, complicações hemorrágicas, e necessidade de reintervenção cirúrgica. Nossos achados coincidem com os de Colleoni et al., ${ }^{10}$ que relataram não haver diferença na deiscência da ferida, e nas taxas de infecção profunda e de reintervenção cirúrgica entre os pacientes que fizeram uso de AAS e de rivaroxabana. Em contradição com os nossos achados, Zou et al. ${ }^{11}$ observaram que o uso de rivaroxabana acarretou uma incidência maior de complicações das feridas e equimoses subcutâneas do que o de AAS. No entanto, os pacientes do estudo de Zou et al. ${ }^{11}$ não apresentaram hemorragia intracraniana ou do trato gastrointestinal, o que coincidiu com os achados do nosso estudo.

\section{Conclusão}

A aspirina e a rivaroxabana apresentaram eficácia comparável na prevenção do TEV, sem aumentar a incidência de complicações da ferida e sangramento após ATJ. Na ATJ primária, o AAS e a rivaroxabana podem ser empregados na prevenção do TEV. Estudos adicionais devem ser realizados com uma população maior, com o objetivo de fornecer resultados mais precisos e informações mais úteis.

\section{Disponibilidade de Dados e Materiais}

O conjunto de dados gerados durante este estudo está disponível com o autor principal, mediante solicitação justificada.

\section{Conflito de Interesses}

Os autores declaram não haver conflito de interesses.

\section{Agradecimentos}

Os autores gostariam de agradecer a Andrew Jonathan Tait, do Departamento de Assuntos Internacionais, por sua ajuda na revisão da língua inglesa deste relatório.

\section{Referências}

1 Bala A, Huddleston JI III, Goodman SB, Maloney WJ, Amanatullah DF. Venous Thromboembolism Prophylaxis After TKA: Aspirin, Warfarin, Enoxaparin, or Factor Xa Inhibitors? Clin Orthop Relat Res 2017;475(09):2205-2213

2 Colwell CW. The ACCP guidelines for thromboprophylaxis in total hip and knee arthroplasty. Orthopedics 2009;32(12, Suppl) 67-73

3 Eikelboom JW, Karthikeyan G, Fagel N, Hirsh J. American Association of Orthopedic Surgeons and American College of Chest Physicians guidelines for venous thromboembolism prevention in hip and knee arthroplasty differ: what are the implications for clinicians and patients? Chest 2009;135(02): 513-520

4 Liu J, Zhao J, Yan Y, Su J. Effectiveness and safety of rivaroxaban for the prevention of thrombosis following total hip or knee replacement: A systematic review and meta-analysis. Medicine (Baltimore) 2019;98(09):e14539

5 Wilson DGG, Poole WEC, Chauhan SK, Rogers BA. Systematic review of aspirin for thromboprophylaxis in modern elective total hip and knee arthroplasty. Bone Joint J 2016;98-B(08): 1056-1061

6 Chen CF, Tsai SW, Wu PK, Chen CM, Chen WM. Does continued aspirin mono-therapy lead to a higher bleeding risk after total knee arthroplasty? J Chin Med Assoc 2019;82(01):60-65

7 Huang HF, Li SS, Yang XT, Xie Q Tian XB. Rivaroxaban versus enoxaparin for the prevention of venous thromboembolism after total knee arthroplasty: A meta-analysis. Medicine (Baltimore) 2018;97(48):e13465

8 Sharrock NE, Gonzalez Della Valle A, Go G, Lyman S, Salvati EA. Potent anticoagulants are associated with a higher all-cause mortality rate after hip and knee arthroplasty. Clin Orthop Relat Res 2008;466(03):714-721

9 Charters MA, Frisch NB, Wessell NM, Dobson C, Les CM, Silverton CD. Rivaroxaban Versus Enoxaparin for Venous Thromboembolism Prophylaxis after Hip and Knee Arthroplasty. J Arthroplasty 2015;30(07):1277-1280

10 Colleoni JL, Ribeiro FN, Mos PAC, Reis JP, Oliveira HR, Miura BK. Venous thromboembolism prophylaxis after total knee arthroplasty (TKA): aspirin vs. rivaroxaban. Rev Bras Ortop 2017;53(01):22-27

11 Zou Y, Tian S, Wang Y, Sun K. Administering aspirin, rivaroxaban and low-molecular-weight heparin to prevent deep venous thrombosis after total knee arthroplasty. Blood Coagul Fibrinolysis 2014;25(07):660-664 
746 Aspirina versus rivaroxabana na prevenção do tromboembolismo venoso Hongnaparak et al.

12 Matharu GS, Kunutsor SK, Judge A, Blom AW, Whitehouse MR. Clinical Effectiveness and Safety of Aspirin for Venous Thromboembolism Prophylaxis After Total Hip and Knee Replacement: A Systematic Review and Meta-analysis of Randomized Clinical Trials. JAMA Intern Med 2020;180(03):376-384

13 Xu J, Kanagaratnam A, Cao JY, Chaggar GS, Bruce W. A comparison of aspirin against rivaroxaban for venous thromboembolism prophylaxis after hip or knee arthroplasty: A metaanalysis. J Orthop Surg (Hong Kong) 2020;28(01): 2309499019896024

14 Yuenyongviwat V, Tuntarattanapong P, Chuaychoosakoon C, Iemsaengchairat $\mathrm{C}$, Iamthanaporn $\mathrm{K}$, Hongnaparak T. Aspirin versus rivaroxaban in postoperative bleeding after total knee arthro- plasty: a retrospective case-matched study. Eur J Orthop Surg Traumatol 2019;29(04):877-881

15 Matharu GS, Garriga C, Whitehouse MR, Rangan A, Judge A. Is Aspirin as Effective as the Newer Direct Oral Anticoagulants for Venous Thromboembolism Prophylaxis After Total Hip and Knee Arthroplasty? An Analysis From the National Joint Registry for England, Wales, Northern Ireland, and the Isle of Man. J Arthroplasty 2020;35(09):2631-2639.e6

16 Kanchanabat B, Stapanavatr W, Meknavin S, Soorapanth C, Sumanasrethakul C, Kanchanasuttirak P. Systematic review and metaanalysis on the rate of postoperative venous thromboembolism in orthopaedic surgery in Asian patients without thromboprophylaxis. Br J Surg 2011;98(10):1356-1364 